\title{
Fisioterapia respiratoria: drenaje postural y evidencia científica
}

\author{
Respiratory physiotherapy: postural drainage and scientific evidence
}

\author{
L. González Doniz, S. Souto Camba, A. López García
}

La fisioterapia respiratoria constituye una de las especialidades con más trayectoria dentro de la fisioterapia, y tiene como finalidad la prevención, el tratamiento y la estabilización de las afecciones respiratorias, ayudando al organismo a utilizar eficazmente todos los mecanismos anatomofuncionales y fisiológicos. Para ello dispone de una serie de procedimientos que se clasifican según sus objetivos, entre ellos, la permeabilización de la vía aérea, la reeducación del patrón ventilatorio y el entrenamiento al esfuerzo ${ }^{1}$.

El drenaje postural forma parte del grupo de procedimientos que tienen como objetivo la permeabilización o aclaramiento de la de la vía aérea y consiste en la adopción de posiciones basadas en la anatomía del árbol bronquial que permiten, por acción de la gravedad, facilitar el flujo de secreciones desde las ramificaciones segmentarias a las lobares, de estas a los bronquios principales y a la tráquea para ser expulsadas, finalmente, al exterior ${ }^{1 \text { and } 2}$. Pretende, por tanto, el drenaje de secreciones bronquiales en aquellas situaciones en las que su aclaramiento esté comprometido, ya sea por una alteración de los mecanismos de la tos que la vuelven ineficaz, por modificaciones en las propiedades reológicas de las secreciones bronquiales, por trastornos en los mecanismos de aclaramiento mucociliar o por defectos estructurales de la vía aérea ${ }^{3}$.

No resulta fácil analizar la evidencia sobre el drenaje postural ya que desde que se empezó a utilizar de forma sistematizada a principios del siglo pasado se ha asociado a la aplicación de percusión y vibración, bajo la denominación de fisioterapia torácica, convencional o estándar. La mayoría de la literatura científica hace pues referencia a estos procedimientos, particularmente al drenaje postural y a la percusión, comparándolos con otros que persiguen el mismo objetivo y tratando de determinar su eficacia. Algunos estudios confirman una mayor producción de esputo en pacientes sometidos a fisioterapia torácica frente a aquellos que no reciben ningún tipo de tratamiento, mostrándose esta especialmente eficaz en las enfermedades hipersecretantes con secreciones de baja adherencia y no parece haber diferencias con otras modalidades de fisioterapia (presión espiratoria positiva, técnica de espiración forzada, ejercicio, drenaje autógeno) en la permeabilización bronquial en niños con fibrosis quística, si bien son pocos los estudios rigurosos que abordan este último aspecto ${ }^{2}$ and 3 .

Pero al lado de los efectos beneficiosos se han puesto en evidencia una serie de efectos adversos del drenaje postural y la percusión que no se han manifestado en la aplicación de otros procedimientos de aclaramiento de la vía aérea tales como el broncoespasmo, la hipoxemia, el incremento en el consumo de oxígeno, el reflujo gastroesofágico en niños, el incremento de la presión intracraneal, la hemorragia intracraneal en grados III y IV en niños pretérmino y las fracturas costales ${ }^{4}$.

Con relación al drenaje postural se ha cuestionado, después de su uso indiscriminado, uno de los posicionamientos clave en su aplicación como es la adopción de posiciones en declive, con la cabeza baja y los posibles efectos secundarios que se derivan de ella.

En este sentido se han estudiado las respuestas cardiovasculares a corto plazo en pacientes sanos comparando una posición en reposo (sentado con las piernas extendidas) con la posición en declive (cabeza abajo $30^{\circ}$ ) observándose pequeñas pero significativas modificaciones en la disminución de la frecuencia cardiaca, la duración diastólica relativa, la presión arterial media y en el índice de tiempo diastólico en la posición en reposo frente a pequeños pero significativos incrementos en el tiempo del ciclo cardiaco, el tiempo de eyección y la duración diastólica absoluta en posición en declive. Así mismo se encontró en esta última posición una pequeña pero significativa caída del índice de viabilidad subendocárdico. Si bien los hallazgos citados tienen consecuencias poco relevantes en los individuos sanos, parecen demostrar que el drenaje postural en posición en declive puede afectar a pacientes receptores de fisioterapia torácica con reserva cardiaca reducida o función baro refleja dañada ${ }^{5}$. 
Ante la necesidad de facilitar la permeabilización de la vía aérea en pacientes inconscientes, donde la aplicación de otros procedimientos de fisioterapia repiratoria estuviesen contraindicados, en un estudio reciente se analizaron las respuestas hemodinámicas agudas en pacientes traumáticos ventilados mecánicamente con función cardiopulmonar estable sometidos a drenaje postural en posición en declive de $30^{\circ}$ comparado con la posición horizontal, analizando la presión arterial, la presión venosa central, la frecuencia cardiaca, la oxigenación y la incidencia de irregularidades cardiacas. Se observaron incrementos significativos en la posición de declive de la presión arterial y la presión venosa central, restaurándose los valores normales a los $10 \mathrm{~min}$ de retomar la posición horizontal. La saturación de oxígeno y la frecuencia cardiaca no sufrieron variaciones y no se presentaron episodios de arritmia o hipoxemia. Los autores concluyen que la posición en declive de $30^{\circ}$ entraña un riesgo mínimo para pacientes traumáticos sin trastorno cardiovascular de base ${ }^{6}$.

Otro efecto deletéreo del drenaje postural en declive de $30^{\circ}$ asociado a percusión es el aumento de reflujo gastroesofágico en niños con fibrosis quística, en los que se observó que el número de episodios por hora se incrementa significativamente durante un programa de fisioterapia que incluye las posiciones en decúbito supino horizontal, decúbito prono con declive de $30^{\circ}$, decúbito lateral derecho e izquierdo con declive de $30^{\circ}$ frente a un programa en posición supina con elevación de $30^{\circ} \mathrm{y}$ decúbito prono, lateral izquierdo y lateral derecho en horizontal ${ }^{7}$.

Así mismo, los pacientes han mostrado una mayor intolerancia y una menor preferencia por el drenaje postural en posición en declive y la percusión con respecto a otros procedimientos como el drenaje autógeno ${ }^{8}$ o la presión espiratoria positiva ${ }^{3}$, mostrando, en definitiva, preferencia por los procedimientos autónomos con respecto a los procedimientos asistidos y dependientes de la intervención.

Las investigaciones sobre los efectos adversos del drenaje postural y la percusión han modificado la práctica de la fisioterapia en los últimos años, evitándose la adopción de posiciones en declive para la movilización de secreciones pero adoptando posiciones para optimizar la ventilación de las diferentes regiones pulmonares (modificando la relación ventilación/perfusión). Se ha teorizado sobre cómo la redistribución de la ventilación podría cambiar la permeabilidad local de la vía aérea, aumentar la ventilación del lado de apoyo y existen evidencias sobre la mayor movilización de secreciones del lado infralateral, contrariamente a los postulados del drenaje postural y la expansión de la vía aérea del lado supralateral $^{9}$.

Dada la alta incidencia y prevalencia de la enfermedad respiratoria y la necesidad de combatir el éxtasis de secreciones bronquiales a partir de los procedimientos de fisioterapia respiratoria se debe seguir profundizando en el conocimiento de los métodos de permeabilización de la vía aérea con estudios de mayor rigor metodológico y que permitan el conocimiento de sus efectos sobre la función cardiorrespiratoria, tanto a corto plazo como a largo plazo, aspecto este último poco estudiado.

\section{Bibliografía}

1. G. Valenza, L. González, M.J. Yuste. Manual de fisioterapia respiratoria y cardiaca. Síntesis, Madrid (2005).

2. J.B. Fink. Positioning versus postural drainage. Respir Care, 47 (2002), pp. 769-777.

3. F.D. McCool, M.J. Rosen. Nonpharmacologic airway clearance therapies: ACCP evidence-based clinical practice guidelines. Chest, 129 (Suppl 1) (2006), pp. S250-S259.

4. D.R. Hess. The evidence for secretion clearance techniques. Cardiopulm Phys Ther J, 13 (2002), pp. 7-22.

5. J.M. Naylor, A.S. McLean, R.C. Heard, A. Avolio. Cardiovascular responses to short-term head-down positioning in healthy young and older adults. Physiother Res Int, 10 (2005), pp. 32-47.

6. G. Hongrattana, P. Reungjui, C.U. Jones. Acute hemodynamic responses to $30^{\circ}$ head-down postural drainage in stable, ventilated trauma patients: A randomized crossover trial. Heart Lung, 43 (2014), pp. 399-405.

7. B.M. Buton, R.G. Heine, A.G. Catto-Smith, P.D. Phelan, A. Olinsky. Postural drainage and gastro-oesophageal reflux in infants with cystic fibrosis. Arch Dis Child, 76 (1997), pp. 148-150.

8. M.P. Mcllwaine, N.M. Lee Son, M.L. Richmond. Physiotherapy and cystic fibrosis: What is the evidence base? Curr Opin Pulm Med, 20 (2014), pp. 613-617.

9. M. Mcllwaine. Physiotherapy and airway clearance techniques and devices. Paediatr Respir Rev, 7 (Suppl 1) (2006), pp. S220-S222. 